\title{
Measurement of the proton scalar polarizabilities at MAMI
}

\author{
Edoardo Mornacchi ${ }^{1 *}$ \\ for the A2 Collaboration \\ ${ }^{1}$ Johannes Gutenberg - Universität Mainz, Germany
}

\begin{abstract}
The electric $\left(\alpha_{\mathrm{E} 1}\right)$ and magnetic $\left(\beta_{\mathrm{M} 1}\right)$ scalar polarizabilities describe the response of the nucleon to an applied electric or magnetic field. They are not only fundamental properties related to the internal structure and dynamics of the nucleon, but they are important also in other areas of physics, such as atomic structure. The values of $\alpha_{\mathrm{E} 1}$ and $\beta_{\mathrm{M} 1}$ quoted by the Particle Data Group were determined using data on the unpolarized differential cross-section of the Compton scattering $\gamma p \rightarrow \gamma p$. The measurement of the beam asymmetry $\Sigma_{3}$, provides an alternative approach to the extraction of the scalar polarizabilities, with different sensitivity and systematics compared to the unpolarized crosssection. This asymmetry was measured recently for the first time below the pion photoproduction threshold by the A2 Collaboration with the Crystal Ball/TAPS experiment at MAMI (Mainz, Germany). A new high precision measurement of both a unpolarized cross-section and beam asymmetry $\Sigma_{3}$ a is ongoing at MAMI and the polarizabilities $\alpha_{\mathrm{E} 1}$ and $\beta_{\mathrm{M} 1}$ will be extracted with unprecedented precision.
\end{abstract}

\section{Introduction}

The scalar polarizabilities $\alpha_{E_{1}}$ and $\beta_{M_{1}}$ characterise the response of a nucleon to an external static electromagnetic field. They not only play a fundamental role in our understanding of the nucleon, but they limit the precision in many other areas of physics. They can be extracted by measuring the differential unpolarized cross section [1] and the beam asymmetry $\Sigma_{3}$ [2] for Compton scattering. The current PDG [3] values for the scalar dipole polarizabilities are $\alpha_{\mathrm{E} 1}=(11.2 \pm 0.4) \times 10^{-4} \mathrm{fm}^{-3}$ and $\beta_{\mathrm{M} 1}=(11.2 \pm 0.4) \times 10^{-4} \mathrm{fm}^{-3}$. These values were previously extracted only from the unpolarized cross-section in different Compton scattering experiments, and the largest data set was obtained by the TAPS collaboration at MAMI [4]. A first measurement of the beam asymmetry $\Sigma_{3}$ below pion threshold was performed by the A2 Collaboration [5].

\section{Experiment}

A new high precision measurement of both unpolarized cross-section and beam asymmetry $\Sigma_{3}$ is ongoing at MAinzer MIkrotron (MAMI) facility [6] in Mainz, Germany. The A2 Collaboration carried out a measurement with a linearly polarized tagged photon beam obtained via coherent bremsstrahlung on a thin diamond radiator. After passing through a collimator,

\footnotetext{
*e-mail: emornacc@uni-mainz.de
} 


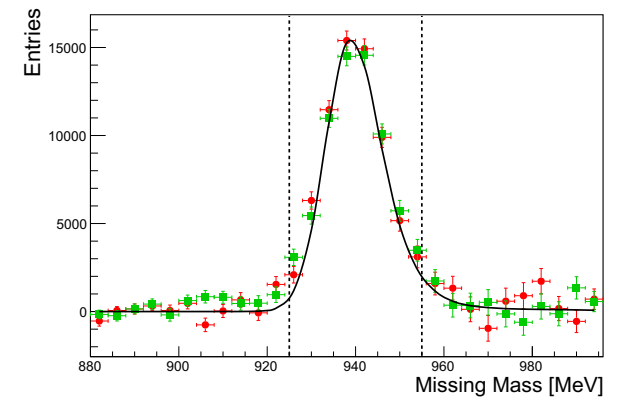

(a)

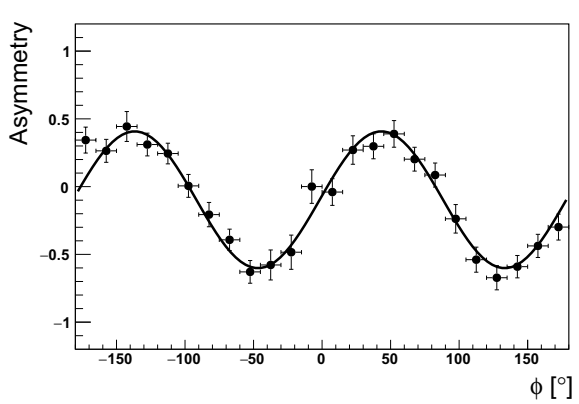

(b)

Figure 1: (a) Preliminary missing mass spectra for $E_{\gamma}=100-120 \mathrm{MeV}$ and $30^{\circ}<\theta<155^{\circ}$ for the two polarization planes with a relative offset of $90^{\circ}$ (red circles and green squares) compared with the Monte Carlo simulated distribution for Compton scattering events (solid black line). (b) Preliminary $\phi$-distribution for $E_{\gamma}=120-140 \mathrm{MeV}$ and $80^{\circ}<\theta<100^{\circ}$ obtained from equation 3 .

the photon beam impinged on a $10 \mathrm{~cm}$ long liquid hydrogen target. The products of the reaction were detected by the Crystal Ball/TAPS setup, covering nearly $97 \%$ of the full solid angle. Further information about the full A2 setup can be found in Refs. [7, 8]. The measurements were performed with two orientations of the photon polarization vector, resulting in a relative shift of $90^{\circ}$ in respect to each other. In order to select Compton scattering events on proton, a single neutral hit in the Crystal Ball was required and events with an outgoing photon angle $\theta>30^{\circ}$ were selected. Additionally, to remove the background due to random coincidences in the Crystal Ball, a time-coincidence between the scattered photon and the hit in the tagger was required and the remaining random background was subtracted. Furthermore, in order to remove events originating from the target cell, the same analysis procedure was applied to a dataset taken with an empty target, and the obtained contribution was scaled and subtracted. A preliminary analysis of $\sim 70 \%$ of the total dataset is already performed, resulting in more than $6.5 \times 10^{5}$ Compton scattering events.

Figure 1a shows the corresponding missing mass spectra, calculated via

$$
m_{m i s s}=\sqrt{\left(E_{\gamma_{i}}+m_{p}-E_{\gamma_{f}}\right)^{2}-\left(\overrightarrow{p_{\gamma_{i}}}-\overrightarrow{p_{\gamma_{f}}}\right)^{2}}
$$

assuming an incoming photon $q_{i}=\left(E_{\gamma_{i}}, \overrightarrow{p_{\gamma_{i}}}\right)$ impinges on the proton $k_{i}=\left(m_{p}, 0,0,0\right)$ and results in a scattered photon $q_{f}=\left(E_{\gamma_{f}}, \overrightarrow{p_{f}}\right)$. For a Compton scattering event $m_{\text {miss }}=m_{p}$. The good agreement between both the datasets and the Monte Carlo simulation indicates absence of significant systematic effects as well as a low background contamination. The two dotted lines show the cut applied on the missing mass value in the analysis.

\section{Beam asymmetry $\Sigma_{3}$}

The use of a linearly polarized photon beam with an unpolarized target introduces azimuthal dependence to the cross section that can be described as

$$
\sigma_{\mathrm{pol}}=\sigma_{\mathrm{unpol}}\left(1+\delta_{l} \Sigma_{3} \cos (2 \phi)\right)
$$


where $\phi$ is the angle between the polarization and the scattering planes, $\sigma_{\text {unpol }}$ is the unpolarized contribution to the cross section, $\delta_{l}$ is the degree of linear polarization of the incoming photon, and $\Sigma_{3}$ defines the amplitude of the $\cos (2 \phi)$ modulation. To account for the variation of the polarization degree along the beamtime for the two datasets with different orientation of the polarization plane, the number of Compton scattering events was weighted with the corresponding value of the linear polarization in the equation for $\Sigma_{3}$ derived from equation 2

$$
\Sigma_{3} \cos \left(2\left(\phi+\phi_{0}\right)\right)=\frac{\sigma_{\|}-\sigma_{\perp}}{\delta_{\perp} \sigma_{\|}-\delta_{\|} \sigma_{\perp}}=\frac{N_{\|}-N_{\perp}}{\delta_{\perp} N_{\|}-\delta_{\|} N_{\perp}},
$$

where $\delta_{\|(\perp)}$ and $\mathrm{N}_{\|(\perp)}$ represent respectively the degree of linear polarization and the event rates for the two polarization settings, and $\phi_{0}$ takes in account a possible deviation of the polarization planes from the expected position. Figure $1 \mathrm{~b}$ shows a sample of $\phi$ distribution in which the $\cos (2 \phi)$ modulation can be clearly identified, according to equations 2 and 3 . The beam asymmetry $\Sigma_{3}$ was extracted by fitting the $\phi$ distributions (see figure 1b) and equating to $\sin (\Delta \phi) / \Delta \phi \Sigma_{3} \cos \left(2\left(\phi+\phi_{0}\right)\right.$ ) in equation 3 (where the prefactor $\sin (\Delta \phi) / \Delta \phi$ accounts for the width of the bins in $\phi)$.

\section{Conclusion}

A new high precision Compton scattering experiment was performed with the A2 setup at the MAMI facility in Mainz. The data were acquired in the first half of this year. A preliminary extraction of both the beam asymmetry $\Sigma_{3}$ and the unpolarized cross section shows very promising results, both in terms of statistics and systematics. The analysis of the collected data is ongoing. Once the full dataset will be available, we expect to have more than $1 \times 10^{6}$ Compton events. This will results in an improvement in statistics by a factor of about 5 compared to the data published in 2017 from the A2 collaboration [5], and it will be the biggest Compton dataset in the relevant energy range.

\section{References}

[1] D. Babusci, G. Giordano, A.I. L’vov, G. Matone, A.M. Nathan, Phys. Rev. C 58, 1013 (1998)

[2] N. Krupina, V. Pascalutsa, Phys. Rev. Lett. 110, 262001 (2013)

[3] M. Tanabashi et al. (Particle Data Group), Phys. Rev. D 98, 030001 (2018)

[4] V. Olmos de Leon, et al., Eur. Phys. J. A 10, 207 (2001)

[5] V. Sokhoyan, et al., Eur. Phys. J. A 53, 14 (2017)

[6] K.-H. Kaiser, et al., Nucl. Instrum. Methods 593, 159 (2008)

[7] A. Starostin, et al., Phys. Rev. C 64, 055205 (2001)

[8] R. Novotny, IEEE Trans. Nucl. Sci. 38, 379 (1991) 